\title{
Significant risk factors for malignant transformation of ovarian endometrioma during dienogest treatment: a case report and retrospective study
}

Michiko Honda, Wataru Isono*, Akira Tsuchiya, Ako Saito, Hiroko Tsuchiya, Reiko Matsuyama, Akihisa Fujimoto and Osamu Nishii

\begin{abstract}
Background: To determine the prevalence of and risk factors for malignant transformation of ovarian endometrioma during dienogest therapy, which is very rare, we examined multiple cases of malignant transformation of ovarian endometrioma during dienogest therapy and performed a multivariate analysis of the records in our hospital.

Methods: The medical records of 174 patients who underwent DNGT for the treatment of OMA from June 1 , 2011, to May 31, 2018, were reviewed retrospectively with the approval of the Human Ethical Committee of the University of Teikyo Hospital. And we provided one representative case of MT with obtaining written informed consent. To assess the effects of six representative factors, including advanced age, parity, surgical history, and endometrial cyst characteristics (including 3 factors), on the possibility of malignant transformation, we performed a multivariate logistic regression analysis.

Results: Of the 174 cases, 4 were diagnosed with malignant transformation, and these cases are reported. In the multivariate analysis, advanced age $(P=0.0064)$, nullipara $(P=0.0322)$, and enlargement $(P=0.0079)$ showed significant differences for malignant transformation occurrence. All 4 malignant transformation cases were among the 19 patients who had all of these 3 factors.
\end{abstract}

Conclusions: For a more accurate determination of the treatment approach, a larger sample size will be needed to determine the risk factors for malignant transformation during dienogest therapy.

Keywords: Endometrioma, Multivariate analysis, Progestin, Retrospective study, Tumorigenic transformation

\section{Background}

Endometriosis is among the most common gynecological diseases and is known to affect $2-10 \%$ of women of reproductive age $[1,2]$. This disease most commonly affects the ovaries, forming a cystic mass called an ovarian endometrioma (OMA) [3], and is diagnosed by an outpatient ultrasound examination. In many cases, magnetic resonance imaging (MRI) is needed for a definitive diagnosis [4]. Because endometriosis is frequently associated

\footnotetext{
* Correspondence: tetuken2010@gmail.com

Department of Obstetrics and Gynaecology, University Hospital Mizonokuchi, Teikyo University School of Medicine, Kanagawa, 5-1-1, Kawasaki, Takatsu-ku, Futago, Kanagawa 213-8507, Japan
}

with infertility and pelvic pain [5], laparoscopic surgery or drug treatment is often performed for symptom relief [6]. The use of dienogest therapy (DNGT), a drug-based treatment, has recently expanded because DNGT has a curative effect in terms of pelvic pain [7] and is expected to reduce the size of an OMA [8]. This therapy can also reduce postoperative lesion recurrence and extend the pain-free period [9]. Thus, dienogest is a new and effective drug. However, several adverse effects, including uterine bleeding, have been reported [10]. Among these side effects, some cases of malignant transformation (MT) during DNGT have been reported [11], although MT during DNGT is very rare. However, a detailed analysis of 
this risk factor has not been performed. For this reason, in addition to the present report of several cases of MT during DNGT in our hospital, we have performed a multivariate analysis of the risk factors for MT during DNGT.

Recent studies have indicated that endometrioid carcinoma and clear cell carcinoma are associated with OMA [12]. Ovarian endometrioma is a subtype of endometriosis, affecting $17-44 \%$ of women with endometriosis [13]. In most cases, this disease is diagnosed as endometrioma by an outpatient ultrasound examination [4], and laparoscopic surgeries are often performed to treat pelvic pain and subfertility [6]. In a previous report, these cancers reportedly developed in $0.72 \%$ of women with a previous diagnosis of OMA who had been managed expectantly [14]. Conversely, some reports showed that a certain ratio of patients with ovarian cancers have a history of endometriosis [15, 16]. However, no significant relationship between previous treatments with hormone drugs (or surgery) and the risk of MT has been validated $[17,18]$. Therefore, our analysis may provide new insight into preventing future cases of MT of OMA.

\section{Methods}

\section{Data collection}

This study was reviewed and approved by the Human Ethics Committee of the University of Teikyo Hospital (trial registration number 18-233). The de-identified medical records of 185 female patients who received dienogest for the treatment of OMA between June 1, 2011, and May 31, 2018, were reviewed retrospectively with the approval of the Human Ethical Committee of the University of Teikyo Hospital. And we provided one representative case of MT with obtaining written informed consent. In this study, we included patients with and without surgery for OMA. Of the 185 patients, 11 patients were excluded because of insufficient data. Among the 174 remaining cases were 4 cases with MT of OMA, and the following data were collected: (1) the size of the OMA before and after starting DNGT (when the patients had bilateral OMA, we adopted the larger OMA from the right or left side), (2) the patient's parity (nulliparous or multiparous patient), (3) the patient's age at the time of starting DNGT, (4) the location of the endometrial cyst before starting DNGT (unilateral or bilateral), and (5) whether surgery was performed. In this study, we focused mostly on the change in the cyst size. In addition, 174 cases were classified into the following three groups: (1) "reduction," defined as cases in which the cyst size showed a decline of $30 \%$ or more; (2) "enlargement," defined as cases in which the cyst size showed an increase of $30 \%$ or more or in which a new cyst appeared after surgery; and (3) "no change," which included the remaining cases.
Usually, when treating patients with endometriosis, we use the revised American Society for Reproductive Medicine (rASRM) score to classify the severity [19]. However, due to insufficient data, we could not include the rASRM score in the multivariate analysis. Because not all patients underwent surgery, we were able to obtain data regarding the rASRM score for only 73 cases $(42 \% ; n=73$ of 174$)$. When an ovarian endometrioma is detected, the severity of endometriosis is regarded as moderate or severe, meaning that the severity of endometriosis is at least stage 3 among four stages. Therefore, all patients in this analysis had a certain level of endometriosis severity. Additionally, we did not analyze the treatment period of dienogest, because DNGT was generally used for the long term and was continued until menopause in some cases [20].

\section{Statistical analysis}

We performed a multivariate logistic regression analysis to assess the influence of the following six factors on the possibility of MT: (1) "advanced age," defined as patients who started DNGT at 41 years of age or older; (2) nullipara; (3) "large cyst," defined as patients whose cyst sizes were $8 \mathrm{~cm}$ or larger at the start of DNGT; (4) "surgical history," defined as patients who underwent surgery for OMA; (5) bilateral cysts; and (6) enlargement. These statistical analyses were performed using JMP version 12 for Windows (SAS Institute, Inc., Tokyo, Japan). The data are presented as the means \pm standard deviations. A $P$ value less than 0.05 was considered statistically significant.

\section{Case presentation}

A 43-year-old Japanese woman, gravida 0, para 0, was referred to our hospital because of aggravated dysmenorrhea and hypermenorrhea. An ovarian cyst had been noted in this patient 3 years before her visit to our hospital, and she had dysmenorrhea beginning at approximately 30 years of age. MRI that was performed before her visit to our hospital suggested a left endometrioma that was $7 \mathrm{~cm}$ in diameter and a right endometrioma that was $3 \mathrm{~cm}$ in diameter, with no solid component in either endometriotic cyst. Bilateral laparoscopic cystectomy (LC) was performed with the patient under general anesthesia, and the diameters of the right and left endometriomas were 3 $\mathrm{cm}$ and $7 \mathrm{~cm}$, respectively (Fig. $1 \mathrm{a}$ and b). After both ovaries were subjected to a surgical procedure, including complete Douglas pouch obliteration, to release them from their severe pelvic adhesion, the left endometrial cyst was completely excised, and the right endometrial cyst was ablated. The rASRM score was 81 points, and this case was diagnosed as stage IV endometriosis. The ovarian cyst did not contain a solid component (Fig. 1c), and pathological examination showed a left endometriotic cyst and no malignancy (Fig. 1d). After surgery, DNGT was 

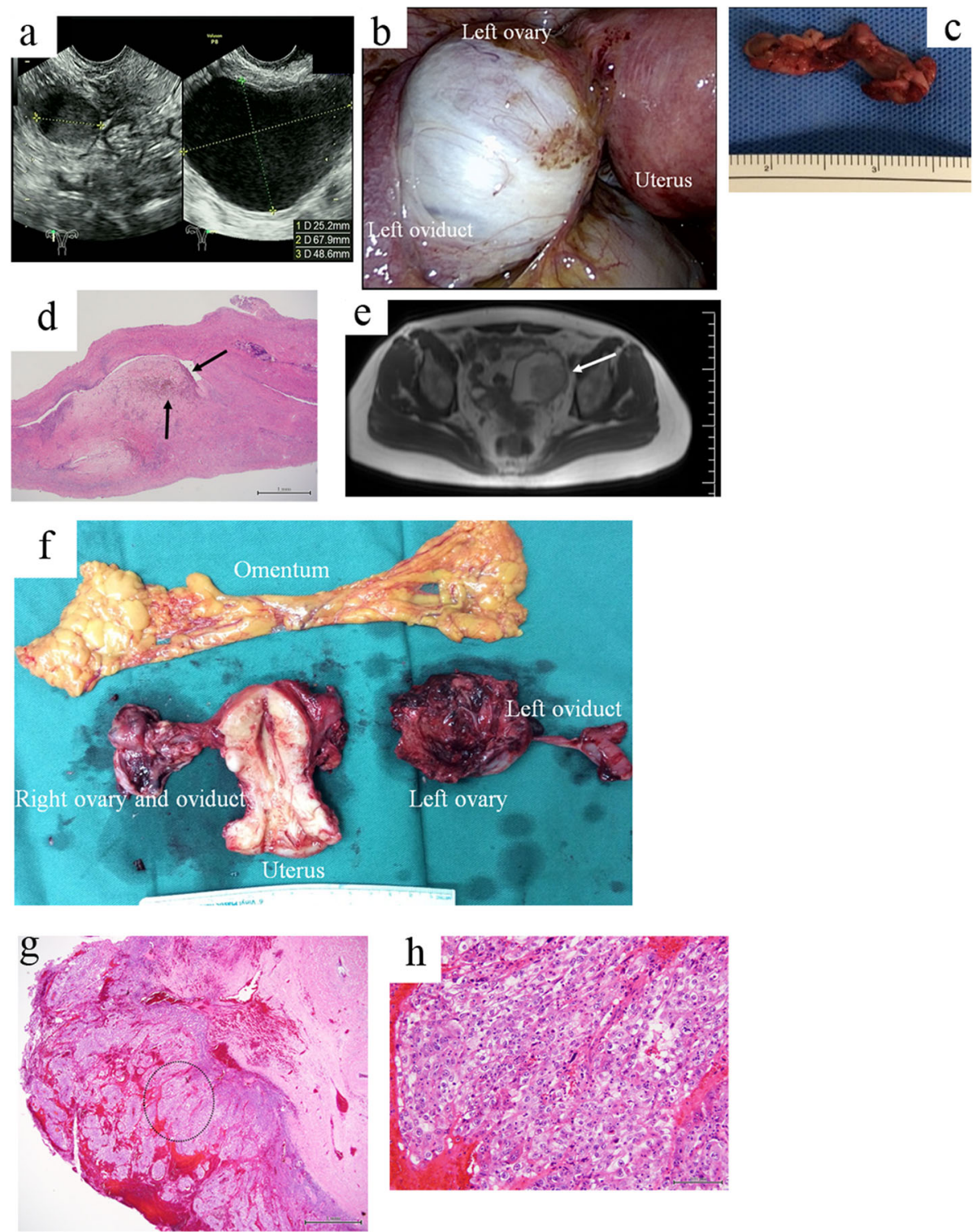

Fig. 1 Images and findings of reported case of malignant transformation. a The sizes of the left and right cysts were $7 \mathrm{~cm}$ and $3 \mathrm{~cm}$ in diameter, respectively, and the cysts were diagnosed by transvaginal ultrasound examination 1 day before surgery. $\mathbf{b}$ Intraoperative image of the left ovarian endometrioma (OMA). The ovarian cyst contains chocolate-like fluid. c Gross appearance of the left ovary at laparoscopic cystectomy (LC). The ovarian cyst does not contain a solid component. $\mathbf{d}$ Pathological findings of the left OMA (H\&E staining, conventional light microscopy at a magnification of $\times 20$ ). Abundant hemosiderin-laden macrophages were present in the cyst wall, as indicated by arrows. e T2-weighted magnetic resonance image at diagnosis of malignant transformation. There was a cystic left ovarian mass $(7 \mathrm{~cm}$ in diameter) with a solid component (arrow). One scale $=1 \mathrm{~cm}$. We could not obtain a magnetic resonance image before $L C$, because the patient had undergone a magnetic resonance imaging examination before visiting our hospital. $\mathbf{f}$ Gross appearance of left ovarian carcinoma and other organs removed at surgery for ovarian cancer. The ovarian cyst contains a large solid component with chocolate-like fluid. $\mathbf{g}$, $\mathbf{h}$ Pathological findings of the left ovarian clear cell carcinoma, as indicated by a circle (H\&E stain, original magnification in $\mathbf{g} \times 20$ and $\mathbf{h} \times 200$ ). Tumor cells have eosinophilic cytoplasm and hyalinized stroma

chosen for the prevention of recurrence, and the patient was monitored by transvaginal ultrasound every 6 months.

At 1 year and 1 month after surgery (that is, 1 year after the start of the administration of DNGT), when the patient was 44 years old, a recurrent left-sided OMA that was 6 $\mathrm{cm}$ in diameter with a solid component inside the left ovarian tumor was detected by outpatient transvaginal ultrasound examination. Contrast-enhanced MRI showed that in addition to a 3-cm right endometrioma, there was a 7-cm left endometrioma with a solid component inside the cavity in which MT was strongly suspected (Fig. 1e). In addition, contrast-enhanced computed tomography exhibited numerous para-aortic lymph node enlargements, including a maximum-sized node that was $10 \mathrm{~mm}$ in 
diameter. The serum cancer antigen 125 level, which was $51.4 \mathrm{U} / \mathrm{ml}$ at the time of the first visit to our hospital, was increased to $515 \mathrm{U} / \mathrm{ml}$. Soon after the imaging diagnosis was performed, complete abdominal surgery, including removal of the uterus, bilateral ovaries, omentum, pelvic lymph nodes, and para-aortic lymph nodes, was performed. The final diagnosis was clear cell carcinoma of the left ovary (Fig. 1f, g, h), International Federation of Gynecology and Obstetrics (FIGO) stage 1C1, pT1C1NOM0. After the necessity of chemotherapy was explained to her, she received chemotherapy of $175 \mathrm{mg} / \mathrm{m}^{2}$ paclitaxel and carboplatin (TC; area under the curve, $6 \mathrm{mg} / \mathrm{ml} / \mathrm{min}$ ) for six cycles and was without recurrence during follow-up.

\section{Results}

\section{Patient characteristics}

In the 174 patients with DNGT, the average endometrioma size before the start of therapy, patient age, and observable treatment period were $4.9 \pm 2.2 \mathrm{~cm}, 39.7 \pm 5.9$ years, and $27.3 \pm 21.6$ months, respectively. There were 70 patients with bilateral OMAs, 51 patients with rightsided OMAs, and 53 patients with left-sided OMAs. We were able to divide the 174 patients into 57 "reduction" cases, 70 "enlargement" cases, and 47 "no change" cases. All four cases of MT during DNGT were included in the "enlargement" group. Therefore, when we compared the change in cyst size, 57 "reduction" cases and 47 "no change" cases were treated as similar cases in the subsequent multivariate analysis.

\section{Summary of four malignant transformation cases}

Table 1 lists the characteristics of the four patients who developed ovarian cancer during DNGT. In this study, we included two patients who were referred to our hospital because the possibility of ovarian cancer during DNGT had been noted in other hospitals. Therefore, 2 of 172 patients who underwent DNGT were diagnosed with MT in our hospital, and the probability reached $1.2 \%$. Of four patients, two patients began DNGT after LC. All four patients were nulliparous. The ages at the time of DNGT ranged from 42 to 44 , and the ages at the time of diagnosis of MT ranged from 42 to 46. The duration of DNGT ranged from 9 to 33 months. Two patients had bilateral OMAs, and two patients had a left OMA. Of four cases, three cases of ovarian cancer developed from a left-sided OMA, and one case of ovarian cancer developed from a rightsided OMA after experiencing recurrence or enlargement of the OMA. As shown in Table 1, the ovarian cancers for all four cases were detected in the early stage and were FIGO stage 1C1 (three cases) or 1C3. Complete surgery, including the removal of the uterus, bilateral ovaries, omentum, pelvic lymph nodes, and para-aortic lymph nodes, was performed in two cases (cases 1 and 3). For the remaining two cases, we performed total hysterectomy and bilateral salpingo-oophorectomy (case 4) or left-sided salpingooophorectomy (case 2). For case 2, we performed interval debulking surgery, including the removal of the uterus, right ovary, omentum, pelvic lymph nodes, and para-

Table 1 Summary of four cases

\begin{tabular}{|c|c|c|c|c|}
\hline & Case 1 & Case 2 & Case 3 & Case 4 \\
\hline Patient's parity & Nullipara & Nullipara & Nullipara & Nullipara \\
\hline Age at start of DNGT & 43 years & 44 years & 42 years & 44 years \\
\hline Age at MT & 44 years & 46 years & 42 years & 46 years \\
\hline Cyst size before DNGT & $\begin{array}{l}7 \mathrm{~cm} \text { (left), } 3 \mathrm{~cm} \\
\text { (right) }\end{array}$ & $8 \mathrm{~cm}$ (left) & $4 \mathrm{~cm}$ (left) & $3 \mathrm{~cm}$ (left) \\
\hline Cyst size at MT & $\begin{array}{l}7 \mathrm{~cm} \text { (left), } 3 \mathrm{~cm} \\
\text { (right) }\end{array}$ & $5 \mathrm{~cm}$ (left) & $\begin{array}{l}7 \mathrm{~cm} \text { (left), } 2 \mathrm{~cm} \\
\text { (right) }\end{array}$ & $\begin{array}{l}3 \mathrm{~cm} \text { (left), } 2 \mathrm{~cm} \\
\text { (right) }\end{array}$ \\
\hline Duration of DNGT & 14 months & 31 months & 9 months & 33 months \\
\hline Operation for ovarian carcinoma & $\begin{array}{l}\text { TAH, BSO, OM, PLA, } \\
\text { PALA }\end{array}$ & LSO & $\begin{array}{l}\text { TAH, BSO, OM, PLA, } \\
\text { PALA }\end{array}$ & $\mathrm{TAH}, \mathrm{BSO}, \mathrm{OM}$ \\
\hline Pathology & Clear cell carcinoma & Clear cell carcinoma & Clear cell carcinoma & $\begin{array}{l}\text { Clear cell } \\
\text { carcinoma }\end{array}$ \\
\hline Origin of carcinoma & Left ovary & Left ovary & Left ovary & Right ovary \\
\hline FIGO stage & $1 \mathrm{C} 1$ & $1 C 3$ & $1 \mathrm{Cl}$ & $1 \mathrm{C} 1$ \\
\hline Surgical history (age) & Bilateral LC (43 years) & Left-side LC (40 years) & No & No \\
\hline Chemotherapy & TC for 6 cycles & TC for 3 cycles & TC for 6 cycles & TC for 6 cycles \\
\hline Serum CA 125 at MT & $515 \mathrm{U} / \mathrm{ml}$ & $78.5 \mathrm{U} / \mathrm{ml}$ & $42.4 \mathrm{U} / \mathrm{ml}$ & $46.3 \mathrm{U} / \mathrm{ml}$ \\
\hline
\end{tabular}

Abbreviations: BSO Bilateral salpingo-oophorectomy, CA 125 Cancer antigen 125, DNGT Dienogest therapy, FIGO International Federation of Gynecology and Obstetrics, LC Laparoscopic cystectomy, LSO Left salpingo-oophorectomy, MT Malignant transformation, OM Omentectomy, PALA Para-aortic lymphadenectomy, PLA Pelvic lymphadenectomy, RSO Right salpingo-oophorectomy, TAH Total abdominal hysterectomy, TC Paclitaxel and carboplatin 
aortic lymph nodes, after the completion of three cycles of TC because the pathological diagnosis was clear cell carcinoma. While performing interval debulking surgery, we detected cancer recurrence in the abdominal cavity and diagnosed the cancer as FIGO stage 3C. After some treatments, including irinotecan, bevacizumab, and oral etoposide, the patient died of extensive metastasis and cancerous peritonitis.

\section{Influential factors in malignant transformation}

To determine significant factors associated with the possibility of MT, we compared the following six factors using multivariate logistic regression models (Table 2): (1) advanced age $(n=45),(2)$ nullipara $(n=113)$, (3) large cyst $(n=18)$, (4) surgical history $(n=81)$, (5) bilateral cysts $(n=70)$, and (6) enlargement $(n=70)$. This analysis revealed that advanced age $(P=0.0064)$, nullipara $(P=0.0322)$, and enlargement $(P=0.0079)$ were significant factors for MT. In particular, all four MT cases were included among 19 patients who had the aforementioned three factors. Therefore, if the patients had these three factors, the possibility of MT reached more than $20 \%$ when choosing DNGT.

\section{Discussion}

Currently, DNGT is being used for patients with symptomatic endometriosis with or without surgery [21]. This progestin agent is reported to decrease the risk of recurrence by inhibiting the growth of endometriotic tissue $[21,22]$. This mechanism can also theoretically prevent MT [23], and reported cases of MT are very rare [24]. However, in our hospital, we had four cases in which ovarian carcinoma was diagnosed during DNGT for

Table 2 Identification of influential factors for malignant transformation

\begin{tabular}{llll}
\hline & Number & Probability & $P$ value \\
\hline Advanced age & 94 & $4.3 \%$ & 0.0064 \\
Nullipara & 113 & $3.5 \%$ & 0.0322 \\
Large cyst & 18 & $5.6 \%$ & 0.1964 \\
Bilateral cysts & 70 & $4.3 \%$ & 0.3231 \\
Surgical history & 81 & $2.5 \%$ & 0.1814 \\
Enlargement & 70 & $5.7 \%$ & 0.0079 \\
Total & 174 & $2.3 \%$ &
\end{tabular}

A multivariate analysis of 174 patients with DNGT was performed to examine the influence of six representative factors that could be collected before starting DNGT, as follows: (1) "advanced age," defined as patients who started DNGT at 41 years of age or older; (2) nullipara; (3) "large cyst," defined as patients whose cyst sizes were $8 \mathrm{~cm}$ or larger at the start of DNGT; (4) "surgical history," defined as patients who underwent surgery for the OMA; (5) bilateral cysts; and (6) enlargement. The number of patients with each factor, the probability of MT, and the $P$ values are shown. "Advanced age," "nullipara," and "enlargement" were identified as significant risk factors for MT. "Large cyst," "bilateral cysts," and "surgical history" were not significant risk factors Abbreviations: DNGT dienogest therapy, MT malignant transformation, OMA ovarian endometrioma
OMA. MT in two of the four cases was detected entirely in our hospital, and the probability of MT after starting DNGT was $1.2 \%(n=2$ of 172$)$. This probability was similar to the ability of endometriosis to transform into malignancy, which has been reported to be approximately $1 \%$ of OMAs in previous studies [14]. In this study, we extracted the details of the patients' clinical histories. Because all four patients were over 40 years old and nulliparous, we hypothesized that there were several risk factors, including the patient's age and parity, for using DNGT. To verify the significant influence of risk factors on MT, a multivariate analysis of six factors extracted before starting DNGT was performed (Table 2). Of the six factors, advanced age, nullipara and increased size and recurrence showed significant increases in the probability of MT. In contrast to a previous study in which the association between spontaneous pregnancy and the disease progression of endometriosis was unclear [25], these results were roughly consistent with those of past reports [26, 27]. In particular, 19 patients who had all three factors showed a probability of over $20 \%$ for the occurrence of ovarian carcinoma (21.1\%, $n=4$ of 19). Because the sample number was very small, the result for this extracted highrisk group might be coincidental. Therefore, a larger sample size will be needed to determine the risk factors for MT. However, in contrast to previous studies, large cyst [26-28] and surgical history [29] did not show a significantly higher probability of MT. "Bilateral cysts" were also not significant. These multivariate analysis results presented the possibility that the characteristics of the patient have a stronger influence on MT than the characteristics and treatment approach of the OMA itself. Because two cases among the four cases of MT exhibited recurrences of OMA after LC and because the other two cases exhibited cyst size enlargement after starting DNGT, careful observation of the cyst size is important, regardless of the use of surgical treatment. However, similar to previous reports [11], in our hospital, we could not prevent the two cases of MT (Table 1, case 1 and case 2 ) by follow-up ultrasound examination at an interval of 6 months.

\section{Conclusions}

Although DNGT seems to be effective in controlling OMA, careful observation of the cyst size is needed. Because the probability for ovarian carcinoma occurrence could exceed $20 \%$ in some cases, particularly for nulliparous women of advanced age, a more individualized choice of therapy might be needed. For a more accurate determination of the treatment approach, a larger sample size will be needed to determine the risk factors for MT.

Abbreviations

DNGT: Dienogest therapy; FIGO: International Federation of Gynecology and Obstetrics; LC: Laparoscopic cystectomy; MRI: Magnetic resonance imaging; MT: Malignant transformation; OMA: Ovarian endometrioma; rASRM: Revised 
American Society for Reproductive Medicine score; TC: Paclitaxel and carboplatin

\section{Acknowledgements}

This research was supported by the Mizonokuchi Hospital of Teikyo University in regard to the provision of medical information. The author(s) disclose the receipt of financial support from the Japan Society for the Promotion of Conventions for the research, authorship, and/or publication of this article.

\section{Authors' contributions}

$\mathrm{MH}$ contributed mainly by collecting data. WI analyzed data and wrote the manuscript. AT and ON supervised the whole study. AT, AS, RM, and HT performed all operations. AF and ON determined the methods of all operations and supervised all medical procedures. All authors read and approved the final manuscript.

\section{Funding}

The authors declare that no funding was received for this study.

\section{Availability of data and materials}

The authors agree to make all data of this study freely available.

\section{Ethics approval and consent to participate}

This retrospective study was approved by the Institutional Review Board of Teikyo University and we provided one representative case of MT with obtaining written informed consent. The study registry number, registry name, and registration date are 18-233, Clinical outcomes and carcinogenic risk of ovarian endometriomas: a retrospective analysis, and 20 March 2019, respectively.

\section{Consent for publication}

Written informed consent was obtained from four representative patients, including in Table 1, for publication of all images. The retrospective analysis of 185 patients was approved by the Human Ethical Committee of the University of Teikyo Hospital (Trial registration number: 18-233). A copy of the written consent form is available for review by the Editor-in-Chief of this journal.

\section{Competing interests}

The authors declare that they have no competing interests.

Received: 20 June 2019 Accepted: 16 August 2019

Published online: 22 October 2019

\section{References}

1. Huang JQ, Lathi RB, Lemyre $M$, Rodriguez $H E$, Nezhat $\mathrm{CH}$, Nezhat $\mathrm{C}$. Coexistence of endometriosis in women with symptomatic leiomyomas. Fertil Steril. 2010:94(2):720-3.

2. Khan KN, Kitajima M, Hiraki K, Fujishita A, Sekine I, Ishimaru T, et al. Changes in tissue inflammation, angiogenesis and apoptosis in endometriosis, adenomyosis and uterine myoma after GnRH agonist therapy. Hum Reprod. 2010:25(3):642-53.

3. Vigano P, Parazzini F, Somigliana E, Vercellini P. Endometriosis: epidemiology and aetiological factors. Best Pract Res Clin Obstet Gynaecol. 2004:18(2):177-200.

4. Bazot M, Darai E. Diagnosis of deep endometriosis: clinical examination, ultrasonography, magnetic resonance imaging, and other techniques. Fertil Steril. 2017;108(6):886-94.

5. Sinaii N, Plumb K, Cotton L, Lambert A, Kennedy S, Zondervan K, et al. Differences in characteristics among 1,000 women with endometriosis based on extent of disease. Fertil Steril. 2008;89(3):538-45.

6. Singh SS, Suen MW. Surgery for endometriosis: beyond medical therapies. Fertil Steril. 2017;107(3):549-54

7. Kim SA, Um MJ, Kim HK, Kim SJ, Moon SJ, Jung H. Study of dienogest for dysmenorrhea and pelvic pain associated with endometriosis. Obstet Gynecol Sci. 2016;59(6):506-11.

8. Schindler AE. Dienogest in long-term treatment of endometriosis. Int J Womens Health. 2011:3:175-84.

9. Romer T. Long-term treatment of endometriosis with dienogest: retrospective analysis of efficacy and safety in clinical practice. Arch Gynecol Obstet. 2018;298(4):747-53.
10. Park SY, Kim SH, Chae HD, Kim CH, Kang BM. Efficacy and safety of dienogest in patients with endometriosis: a single-center observational study over 12 months. Clin Exp Reprod Med. 2016;43(4):215-20.

11. Yoshino O, Minamisaka T, Ono Y, Tsuda S, Samejima A, Shima T, et al. Three cases of clear-cell adenocarcinoma arising from endometrioma during hormonal treatments. J Obstet Gynaecol Res. 2018;44(9):1850-8.

12. Melin A, Sparen P, Persson I, Bergqvist A. Endometriosis and the risk of cancer with special emphasis on ovarian cancer. Hum Reprod. 2006;21(5): 1237-42.

13. Redwine DB. Ovarian endometriosis: a marker for more extensive pelvic and intestinal disease. Fertil Steril. 1999:72(2):310-5.

14. Kobayashi H, Sumimoto K, Moniwa N, Imai M, Takakura K, Kuromaki T, et al. Risk of developing ovarian cancer among women with ovarian endometrioma: a cohort study in Shizuoka, Japan. Int J Gynecol Cancer. 2007:17(1):37-43.

15. Boussios S, Attygalle A, Hazell S, Moschetta M, McLachlan J, Okines A, et al. Malignant ovarian germ cell tumors in postmenopausal patients: the Royal Marsden experience and literature review. Anticancer Res. 2015;35(12):6713-22.

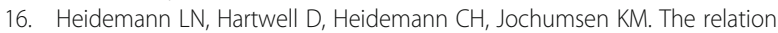
between endometriosis and ovarian cancer - a review. Acta Obstet Gynecol Scand. 2014;93(1):20-31.

17. Kobayashi H, Sumimoto K, Kitanaka T, Yamada Y, Sado T, Sakata M, et al. Ovarian endometrioma-risks factors of ovarian cancer development. Eur J Obstet Gynecol Reprod Biol. 2008;138(2):187-93.

18. Thomsen LH, Schnack TH, Buchardi K, Hummelshoj L, Missmer SA, Forman $A$, et al. Risk factors of epithelial ovarian carcinomas among women with endometriosis: a systematic review. Acta Obstet Gynecol Scand. 2017;96(6):761-78.

19. American Society for Reproductive Medicine. Revised American Society for Reproductive Medicine classification of endometriosis: 1996. Fertil Steril. 1997:67(5):817-21.

20. Neriishi K, Hirata T, Fukuda S, Izumi G, Nakazawa A, Yamamoto N, et al. Long-term dienogest administration in patients with symptomatic adenomyosis. J Obstet Gynaecol Res. 2018;44(8):1439-44.

21. Bedaiwy MA, Allaire C, Alfaraj S. Long-term medical management of endometriosis with dienogest and with a gonadotropin-releasing hormone agonist and add-back hormone therapy. Fertil Steril. 2017;107(3):537-48.

22. Lee $K H$, Jung $Y W$, Song SY, Kang BH, Yang JB, Ko YB, et al. Comparison of the efficacy of diegnogest and levonorgestrel-releasing intrauterine system after laparoscopic surgery for endometriosis. J Obstet Gynaecol Res. 2018; 44(9):1779-86.

23. Del Pup L, Berretta M. As dienogest effectively suppresses endometriosis, could it also reduce endometriosis-associated ovarian cancers? A further motivation for long-term medical treatment. WCRJ. 2015:2(2):e526.

24. Takagi H, Takata E, Sakamoto J, Fujita S, Takakura M, Sasagawa T. Malignant Transformation of an ovarian endometrioma during endometriosis treatment: a case report. Case Rep Obstet Gynecol. 2018;2018:6210172.

25. Leeners B, Damaso F, Ochsenbein-Kolble N, Farquhar C. The effect of pregnancy on endometriosis-facts or fiction? Hum Reprod Update. 2018; 24(3):290-9.

26. Kadan Y, Fiascone S, McCourt C, Raker C, Granai CO, Steinhoff M, et al. Predictive factors for the presence of malignant transformation of pelvic endometriosis. Eur J Obstet Gynecol Reprod Biol. 2015;185:23-7.

27. He ZX, Shi HH, Fan QB, Zhu L, Leng JH, Sun DW, et al. Predictive factors of ovarian carcinoma for women with ovarian endometrioma aged 45 years and older in China. J Ovarian Res. 2017;10(1):45.

28. Sayasneh A, Tsivos D, Crawford R. Endometriosis and ovarian cancer: a systematic review. ISRN Obstet Gynecol. 2011;2011:140310.

29. Taniguchi F. New knowledge and insights about the malignant transformation of endometriosis. J Obstet Gynaecol Res. 2017;43(7):1093-100.

\section{Publisher's Note}

Springer Nature remains neutral with regard to jurisdictional claims in published maps and institutional affiliations. 